\title{
М. М. Сиражудинов
}

\section{Операторные оценки усреднения обобщенных уравнений Бельтрами}

В работе, при помощи асимптотических методов, получены операторные оценки усреднения задачи Римана-Гильберта для обобщенного уравнения Бельтрами с быстро колеблющими $\varepsilon$-периодическими коэффициентами.

Библиография: 10 названий.

Operator estimates for homogenization of the Riemann-Hilbert problem for the generalized Beltrami equation with rapidly oscillating $\varepsilon$-periodic coefficients are considered. Asymptotic methods of homogenization are used.

Bibliography: 10 items.

Ключевые слова: уравнение Бельтрами, усреднение, асимптотические методы

Keywords: Beltrami equation, homogenization, method of asimptotic expansions

\section{1. Обозначения}

$\mathbb{R}^{2}$ - плоскость, $Q \subset \mathbb{R}^{2}$ - ограниченная односвязная область с кусочно гладкой границей, $\bar{Q}$ - замыкание области $Q$.

$\partial_{\bar{z}}=2^{-1}\left(\mathscr{D}_{1}+i \mathscr{D}_{2}\right), \quad \partial_{z}=2^{-1}\left(\mathscr{D}_{1}-i \mathscr{D}_{2}\right), \quad \mathscr{D}_{j}=\frac{\partial}{\partial x_{j}}, \quad j=1,2$.

$i$ - мнимая единица.

$L_{2}(Q ; \mathbb{C})$ - пространство Лебега комплекснозначных квадратично суммируемых функций. Символ $\mathbb{C}$ (здесъ и далее) в обозначении пространства означает такэе, что это пространство есть линейное пространство над полем действительных чисел $\mathbb{R}$. Скалярное произведение в $L_{2}(Q ; \mathbb{C})$ дается равенством:

$$
(u, v)_{L_{2}(Q ; \mathbb{C})}=\operatorname{Re} \int_{Q} u \bar{v} d x, \quad u, v \in L_{2}(Q ; \mathbb{C}),
$$

где $\bar{v}-$ комплексно сопряженная $v$ функция.

Соответствие $L_{2}(Q ; \mathbb{C}) \ni u=u_{1}+i u_{2} \mapsto\left(u_{1}, u_{2}\right)=U \in\left(L_{2}(Q)\right)^{2}$ есть изоморфизм, причем $(U, V)_{\left(L_{2}(Q)\right)^{2}}=(u, v)_{L_{2}(Q ; \mathbb{C})}$.

$W_{p}^{k}(Q ; \mathbb{C})$ - пространство Соболева комплекснозначных функций.

$\square, \square_{T}-$ квадрат со стороной (длины $T$ ), параллельной оси координат (ячейка периодов). $|\square|=T^{2}-$ площадь квадрата $\square$.

Работа выполнена при финансовой поддержке РФФИ (грант №16-01-00508-а)

(C) М. М. СиРАЖУдинов, 2017 
Периодической будем называть функцию периода $T$ по каждой переменной. $\langle u\rangle-$ среднее значение периодической функции $u$, т.е.

$$
\langle u\rangle=|\square|^{-1} \int_{\square} u(x) d x .
$$

$L_{p}(\square ; \mathbb{C}), W_{p}^{1}(\square ; \mathbb{C}), p \geqslant 1,-$ пространства Лебега и Соболева периодических функций. Скалярное произведение в $L_{2}(\square ; \mathbb{C})$ задает равенство $(u, v)_{L_{2}(\square ; \mathbb{C})}=\operatorname{Re}\langle u \bar{v}\rangle, u, v \in L_{2}(\square ; \mathbb{C})$.

$W_{2}^{-1}(\square ; \mathbb{C})-$ пространство, сопряженное $W_{2}^{1}(\square ; \mathbb{C})$. Пространство, сопряженное $L_{2}(\square ; \mathbb{C})$, отождествляем с $L_{2}(\square ; \mathbb{C})$, что возможно, в силу теоремы Рисса.

$\rightarrow-$ знак слабой сходимости в соответствующем пространстве.

Как известно (см. [1; гл. 1$])$, если $g(x)\left(x \in \mathbb{R}^{2}\right)$ - периодическая функция, $g \in L_{p}(\square ; \mathbb{C}), p \geqslant 1$, то тогда $g\left(\varepsilon^{-1} x\right) \rightarrow\langle g\rangle$ в $L_{p \text { loc }}(Q ; \mathbb{C})$ при $\varepsilon \rightarrow 0$, где $Q-$ произвольная ограниченная область плоскости.

$W_{0}(Q)$ - подпространство $W_{2}^{1}(Q ; \mathbb{C})$, элементы которого удовлетворяют соотношениям:

$$
\left.\operatorname{Re} u\right|_{\partial Q}=0, \quad \int_{Q} \operatorname{Im} u d x=0 .
$$

В случае, когда это не вызывает недоразумений, как уравнение, так и оператор соответствующей краевой задачи обозначаем одним и тем же символом.

$y_{j}=\varepsilon^{-1} x_{j}, j=1 ; 2$, - «быстрые» переменные.

Теория усреднения бурно развивается с середины второй половины прошлого века. Этот раздел дифференциальных уравнений имеет многочисленные приложения в различных разделах физики и механики сплошных сред.

Операторные оценки усреднения дивергентных эллиптических операторов получены в [2]. Собственно термин «операторные оценки усреднения» ввел в обиход В. В. Жиков [3]. Изложение различных аспектов операторных оценок усреднения для дивергентных операторов можно найти в обзорной работе [4].

Наша работа посвящена операторным оценкам усреднения для обобщенных уравнений Бельтрами. Эти уравнения являются недивергентными, поэтому полученные операторные оценки усреднения (см. ниже п. 2.6) отличаются от оценок для дивергентных операторов.

\section{2. Формулировка результатов}

В пунктах 2.1 - 2.5 собраны известные результаты, которые нам понадобятся в дальнейшем.

2.1. Задача Римана - Гильберта. Рассмотрим следующую задачу Римана - Гильберта:

$$
\left\{\begin{array}{l}
A u \equiv \partial_{\bar{z}} u+\mu \partial_{z} u+\nu \partial_{\bar{z}} \bar{u}=f \in L_{2}(Q ; \mathbb{C}), \\
u \in W_{0}(Q),
\end{array}\right.
$$


где коэффициенты $\mu=\mu(x), \nu=\nu(x)$ - измеримые ограниченные комплекснозначные функции, удовлетворяющие условию

$$
\underset{x \in Q}{\operatorname{vraisup}}(|\mu(x)|+|\nu(x)|) \leqslant k_{0}<1,
$$

$k_{0}>0$ - постоянная (константа эллиптичности); $Q-$ ограниченная односвязная область плоскости с кусочно-гладкой границей.

Заметим, что любую равномерно эллиптическую систему двух уравнений первого порядка с действительными непрерывными в $\bar{Q}$ коэффициентами можно представить в виде $(2.1),(2.2)$ (см. [5; $§ 7$, гл. 2 и § 17, гл. 3]).

Как известно (см. $[6,7,8,9])$, задача Римана-Гильберта (2.1) однозначно разрешима для любой правой части из $L_{2}(Q ; \mathbb{C})$, причем имеют место априорные оценки:

$$
\begin{gathered}
\left(1-k_{0}\right)\left\|\partial_{\bar{z}} u\right\|_{L_{2}(Q ; \mathbb{C})}^{2} \leqslant \operatorname{Re} \int_{Q} A u \cdot \overline{\partial_{\bar{z}} u} d x, \\
\left(1-k_{0}\right)\left\|\partial_{\bar{z}} u\right\|_{L_{2}(Q ; \mathbb{C})} \leqslant\|A u\|_{L_{2}(Q ; \mathbb{C})} \leqslant\left(1+k_{0}\right)\left\|\partial_{\bar{z}} u\right\|_{L_{2}(Q ; \mathbb{C})} .
\end{gathered}
$$

Оценки (2.3), (2.4) справедливы для любого элемента $u \in W_{2}^{1}(Q ; \mathbb{C})$, удовлетворяющего условию $\left.\operatorname{Re} u\right|_{\partial Q}=0$, условие на мнимую часть не требуется. Более того они верны и для многосвязных областей.

Выражение

$$
\|u\|_{W_{0}(Q)}=\left\|\partial_{\bar{z}} u\right\|_{L_{2}(Q ; \mathbb{C})}, \quad u \in W_{0}(Q)
$$

задает в подпространстве $W_{0}(Q)$ норму, эквивалентную норме исходного пространства $W_{2}^{1}(Q ; \mathbb{C})$ (см. [6]), поэтому имеют место оценки:

$$
c_{1}\|u\|_{W_{2}^{1}(Q ; \mathbb{C})} \leqslant\|A u\|_{L_{2}(Q ; \mathbb{C})} \leqslant c_{2}\|u\|_{W_{2}^{1}(Q ; \mathbb{C})},
$$

где $c_{1}, c_{2}>0$ - положительные постоянные, зависящие только от постоянной эллиптичности $k_{0}$.

2.2. $G$-сходимость. Обозначим через $\mathscr{A}\left(k_{0} ; Q\right)$ - множество обобщенных операторов Бельтрами $(2.1), \mathscr{A}_{0}\left(k_{0} ; Q\right)$ - подмножество $\mathscr{A}\left(k_{0} ; Q\right)$ операторов Бельтрами (2.1) с $\nu=0$.

ОПреДЕЛЕНИЕ 1. Скажем, что последовательность операторов $\left\{A_{k}\right\}$ из класса $\mathscr{A}\left(k_{0} ; Q\right) G$-сходится в области $Q$ к оператору $A \in \mathscr{A}\left(k_{0} ; Q\right)$ (и будем писать $\left.A_{k} \stackrel{G}{\longrightarrow} A\right)$, если для любого $f \in L_{2}(Q ; \mathbb{C})$ последовательность $u_{k}$ решений задачи $\mathrm{P}-\Gamma: A_{k} u_{k}=f, u_{k} \in W_{0}(Q)$, сходится в $L_{2}(Q ; \mathbb{C})$ к решению задачи $\mathrm{P}-\Gamma: A u=f, u \in W_{0}(Q)$.

$G$-предел определен единственным образом. Как известно (см. [6, 7]) классы $\mathscr{A}\left(k_{0} ; Q\right)$ и $\mathscr{A}_{0}\left(k_{0} ; Q\right)$ являются $G$-компактными.

2.3. Усреднение. Рассмотрим задачу Римана - Гильберта:

$$
\left\{\begin{array}{l}
A_{\varepsilon} u \equiv \partial_{\bar{z}} u_{\varepsilon}+\mu^{\varepsilon} \partial_{z} u_{\varepsilon}+\nu^{\varepsilon} \partial_{\bar{z}} \bar{u}_{\varepsilon}=f \in L_{2}(Q ; \mathbb{C}), \\
u_{\varepsilon} \in W_{0}(Q)
\end{array}\right.
$$


где $0<\varepsilon<1, \mu^{\varepsilon}=\mu\left(\varepsilon^{-1} x\right), \nu^{\varepsilon}=\nu\left(\varepsilon^{-1} x\right) ; \mu(x)=\mu\left(x_{1}, x_{2}\right), \nu(x)=\nu\left(x_{1}, x_{2}\right)$ - измеримые ограниченные комплекснозначные периодические (периода $T$ по каждой переменной) функции, удовлетворяющие условию эллиптичности (2.2) на всей плоскости

$$
\underset{x \in \mathbb{R}^{2}}{\operatorname{vraisup}}(|\mu(x)|+|\nu(x)|) \leqslant k_{0}<1 .
$$

Очевидно, что оператор $A_{\varepsilon}$ принадлежит классу $\mathscr{A}\left(k_{0} ; Q\right)$.

ОПРЕДЕЛЕНиЕ 2. Скажем, что семейство $\left\{A_{\varepsilon}\right\}$ допускает усреднение, если $A_{\varepsilon} \stackrel{G}{\longrightarrow} A \in \mathscr{A}\left(k_{0} ; Q\right)$ при $\varepsilon \rightarrow 0$.

В вопросах усреднения важную роль играет ядро оператора $\mathscr{A}^{*}$, сопряженного оператору периодической задачи:

$$
\mathscr{A} u \equiv \partial_{\bar{z}} u+\mu(x) \partial_{z} u+\nu(x) \partial_{\bar{z}} \bar{u}=f \in L_{2}(\square ; \mathbb{C}), \quad u \in W_{2}^{1}(\square ; \mathbb{C}) .
$$

Сформулируем результаты по периодической задаче, необходимые в дальнейшем.

Теорема 2.1 (См. [6]). Справедливы следующие утверждения:

- Периодическая задача (2.7) фредгольмова.

- Ядро Ker $\mathscr{A}^{*}$ сопряженного оператора $\mathscr{A}^{*}: L_{2}(\square ; \mathbb{C}) \rightarrow W_{2}^{-1}(\square ; \mathbb{C})$ двумерное подпространство $L_{2}(\square ; \mathbb{C})$ (напомним, что наши пространства - пространства над полем $\mathbb{R})$, причем один из базисов $\left\{p_{1}, p_{2}\right\}$ ядра $\operatorname{Ker} \mathscr{A}^{*}$ обладает свойствами

$$
\left\langle p_{1}\right\rangle=1, \quad\left\langle p_{2}\right\rangle=i .
$$

- В случае оператора Бельтрами $(\nu=0)$ имеем: $p_{2}=i p_{1}$.

Сформулируем теорему об усреднении из работы [10].

ТЕОРЕМА 2.2 (ОБ УСРЕДНЕНИИ). Для семейства операторов (2.6):

$$
A_{\varepsilon}=\partial_{\bar{z}}+\mu^{\varepsilon} \partial_{z}+\nu^{\varepsilon} \bar{\partial}_{z},
$$

где $\mu^{\varepsilon}=\mu\left(\varepsilon^{-1} x\right), \nu^{\varepsilon}=\nu\left(\varepsilon^{-1} x\right)$, имеет место усреднение, причем коэффициенты усредненного оператора

$$
A_{0}=\partial_{\bar{z}}+\mu^{0} \partial_{z}+\nu^{0} \bar{\partial}_{z}
$$

постоянные, определяемые равенствами

$$
\mu^{0}=\langle\mu \mathscr{Q}+\bar{\nu} \mathscr{P}\rangle, \quad \nu^{0}=\langle\bar{\mu} \mathscr{P}+\nu \mathscr{Q}\rangle,
$$

где

$$
\mathscr{P}=2^{-1}\left(p_{1}+i p_{2}\right), \quad \mathscr{Q}=2^{-1}\left(\overline{p_{1}}+i \overline{p_{2}}\right),
$$

$\left(p_{1}, p_{2}-\right.$ базисные векторы ядра Ker $\mathscr{A}^{*}$ из теоремы $2.1, \overline{p_{1}}, \overline{p_{2}}-$ комплексно

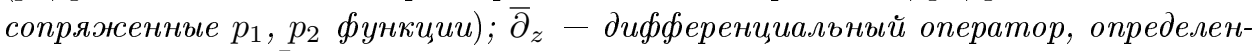
ный равенством $\bar{\partial}_{z} u=\partial_{\bar{z}} \bar{u}$.

В случае уравнения Бельтрами имеем: $\mu^{0}=\left\langle\mu \overline{p_{1}}\right\rangle, \nu^{0}=0$. 
2.4. Задача на ячейке. Рассмотрим следующую периодическую задачу

$$
\left\{\begin{array}{l}
\mathscr{A} N_{j} \equiv \partial_{\bar{z}} N_{j}+\mu \partial_{z} N_{j}+\nu \partial_{\bar{z}} \bar{N}_{j}=\chi_{j}, \\
N_{j} \in W_{2}^{1}(\square ; \mathbb{C}), \quad j=1 ; 2,
\end{array}\right.
$$

где

$$
\chi_{1}=\frac{1}{2}\left(\mu^{0}+\nu^{0}-\mu(x)-\nu(x)\right), \quad \chi_{2}=\frac{i}{2}\left(\mu^{0}-\nu^{0}-\mu(x)+\nu(x)\right) .
$$

Здесь $\mu^{0}, \nu^{0}-$ коэффициенты (2.8) усредненного уравнения. Справедлива следующая

ТЕОРема 2.3 (См. [10]). Периодическая задача (2.9) разрешима и решения представляются в виде

$$
N_{j}+c_{j}, \quad j=1 ; 2,
$$

где $c_{j} \in \mathbb{C}-$ произвольная постоянная, $N_{j} \in W_{2}^{1}(\square ; \mathbb{C})-$ решение $(2.9)$ со средним равным нулю $\left\langle N_{j}\right\rangle=0$.

В случае уравнения Бельтрами $(\nu=0)$, имеем $N_{2}=i N_{1}$.

2.5. Первое приближение к решению задачи (2.6). Пусть правая часть задачи $(2.6) f$ принадлежит пространству $W_{2}^{1}(Q ; \mathbb{C})$ и пусть $u_{\varepsilon}$ - решение задачи Римана-Гильберта (2.6). Первым приближением к решению $u_{\varepsilon}$ задачи (2.6) является функция

$$
u_{1}^{\varepsilon}(x)=u^{0}(x)+\varepsilon\left(\left(N_{1}(y)-i N_{2}(y)\right) \partial_{z} u^{0}(x)+\left(N_{1}(y)+i N_{2}(y)\right) \partial_{\bar{z}} \overline{u^{0}(x)}\right),
$$

где $N_{1}$ и $N_{2}$ - периодические решения задачи на ячейке (см. теорему 2.3), $y=\varepsilon^{-1} x ; u^{0}-$ решение усредненной задачи: $A_{0} u^{0}=f, u^{0} \in W_{0}(Q ; \mathbb{C})$. Оператор $A_{0}$ - эллиптический оператор с постоянными коэффициентами, поэтому $u^{0}$ принадлежит $W_{2}^{2}(Q ; \mathbb{C}) \cap W_{0}(Q)$, ввиду свойств регулярности решений эллиптических уравнений. При этом справедливо соотношение

$$
A_{\varepsilon} u_{1}^{\varepsilon}=f+\varepsilon r_{\varepsilon}
$$

где

$$
\begin{aligned}
& r_{\varepsilon}=\left(N_{1}(y)-i N_{2}(y)\right)\left(\partial_{\bar{z} z}^{2} u^{0}(x)+\mu(y) \partial_{z z}^{2} u^{0}(x)+\nu(y) \partial_{\bar{z} \bar{z}}^{2} \overline{u^{0}(x)}\right)+ \\
& \quad+\left(N_{1}(y)+i N_{2}(y)\right)\left(\partial_{\bar{z} \bar{z}}^{2} \overline{u^{0}(x)}+\mu(y) \partial_{z \bar{z}}^{2} \overline{u^{0}(x)}+\nu(y) \partial_{\bar{z} z}^{2} u^{0}(x)\right), \quad y=\varepsilon^{-1} x,
\end{aligned}
$$

причем невязка $r_{\varepsilon}$ принадлежит пространству $L_{2}(Q ; \mathbb{C})$.

Отметим, что первое приближение $u_{1}^{\varepsilon}$ принадлежит $W_{2}^{1}(Q ; \mathbb{C})$.

Главным результатом работы [10] является следующая

Теорема 2.4. Пусть правая часть $f$ задачи Римана - Гилъберта (2.6) принадлежит пространству $W_{2}^{1}(Q ; \mathbb{C}), Q$ - односвязная область с гладкой (класса $C^{2}$ ) границей, тогда имеют место оценки

$$
\left\|u_{\varepsilon}-u_{1}^{\varepsilon}\right\|_{W_{2}^{1}(Q ; \mathbb{C})} \leqslant c \sqrt{\varepsilon}\|f\|_{W_{2}^{1}(Q ; \mathbb{C})}, \quad\left\|u_{\varepsilon}-u^{0}\right\|_{L_{2}(Q ; \mathbb{C})} \leqslant c \sqrt{\varepsilon}\|f\|_{W_{2}^{1}(Q ; \mathbb{C})},
$$

где с $>0-$ постоянная, независящая от $\varepsilon$ и $f$. 
Первая из оценок (2.13) - оценка разности точного решения задачи (2.6) и первого приближения, а вторая есть оценка скорости сходимости точного решения к решению усредненной задачи.

2.6. Операторные оценки усреднения. Основным результатом работы является следующая

ТЕОРема 2.5. Справедливы следующие операторные оценки усреднения задачи Римана-Гилъберта (2.6)

$$
\begin{gathered}
\left\|\left(A_{\varepsilon}^{-1}-A_{0}^{-1}\right) \partial_{\bar{z}}^{-1}\right\|_{L_{2}(Q ; \mathbb{C}) \rightarrow L_{2}(Q ; \mathbb{C})} \leqslant c \sqrt{\varepsilon} \\
\left\|A_{\varepsilon}^{-1}-A_{0}^{-1}\right\|_{W_{0}(Q) \rightarrow L_{2}(Q ; \mathbb{C})} \leqslant c \sqrt{\varepsilon} \\
\|\left(A_{\varepsilon}^{-1}-A_{0}^{-1}\right) \partial_{\bar{z}}^{-1}-\varepsilon\left(N \partial_{z} A_{0}^{-1} \partial_{\bar{z}}^{-1}+\right. \\
\left.+M \partial_{\bar{z}} \overline{A_{0}^{-1} \partial_{\bar{z}}^{-1}}\right) \|_{L_{2}(Q ; \mathbb{C}) \rightarrow W_{2}^{1}(Q ; \mathbb{C})} \leqslant c \sqrt{\varepsilon}, \\
\left\|A_{\varepsilon}^{-1}-A_{0}^{-1}-\varepsilon\left(N \partial_{z} A_{0}^{-1}+M \partial_{\bar{z}} \overline{A_{0}^{-1}}\right)\right\|_{W_{2}^{1}(Q ; \mathbb{C}) \rightarrow W_{2}^{1}(Q ; \mathbb{C})} \leqslant \sqrt{\varepsilon},
\end{gathered}
$$

где $c>0-$ постояннал; $\partial_{\bar{z}}^{-1}-$ оператор обратный $\kappa$ оператору краевой задачи (2.6) для уравнения Кочи - Римана; $\overline{A_{0}^{-1} \partial_{\bar{z}}^{-1}}$ - оператор, определенный равенством $\overline{A_{0}^{-1} \partial_{\bar{z}}^{-1}} v=\overline{A_{0}^{-1} \partial_{\bar{z}}^{-1} v}$, аналогичный смысл имеет и оператор $\overline{A_{0}^{-1}}$. Здесъ $N=N_{1}(y)-i N_{2}(y), M=N_{1}(y)+i N_{2}(y), y=\varepsilon^{-1} x$, где $N_{1}(y), N_{2}(y)-$ решения задачи на ячейке (см. теорему 2.3$) ; A_{\varepsilon}^{-1}, A_{0}^{-1}$ - обратные операторы $\kappa$ операторам соответствующих задач Римана-Гильберта.

В случае оператора Бельтрами $(\nu=0)$, ввиду теоремы 2.3 , имеем $N=2 N_{1}$, $M=0$. Следовательно, корректоры в (2.16), (2.17) упрощаются и мы имеем для оператора Бельтрами оценки

$$
\begin{gathered}
\left\|\left(A_{\varepsilon}^{-1}-A_{0}^{-1}-\varepsilon N \partial_{z} A_{0}^{-1}\right) \partial_{\bar{z}}^{-1}\right\|_{L_{2}(Q ; \mathbb{C}) \rightarrow W_{2}^{1}(Q ; \mathbb{C})} \leqslant c \sqrt{\varepsilon} \\
\left\|A_{\varepsilon}^{-1}-A_{0}^{-1}-\varepsilon N \partial_{z} A_{0}^{-1}\right\|_{W_{2}^{1}(Q ; \mathbb{C}) \rightarrow W_{2}^{1}(Q ; \mathbb{C})} \leqslant c \sqrt{\varepsilon}
\end{gathered}
$$

ЗАмечАниЕ 1. Отметим, что в операторных оценках (2.14), (2.16) (как это следует из доказательства теоремы 2.5 в $\S 2)$, оператор $\partial_{\bar{z}}^{-1}$ можно заменить на любой обратимый оператор $B$ действующий из $L_{2}(Q ; \mathbb{C})$ на $W_{0}(Q)$. При этом постоянная $c$ будет зависеть от $B$.

\section{3. Доказательство теоремы 2.5}

Пусть правая часть уравнения (2.6) $f$ принадлежит пространству $W_{0}(Q) \subset$ $W_{2}^{1}(Q ; \mathbb{C})$, тогда, ввиду того, что $\|u\|_{W_{0}(Q)}=\left\|\partial_{\bar{z}} u\right\|_{L_{2}(Q ; \mathbb{C})}$ задает в $W_{0}(Q)$ норму, эквивалентную норме пространства $W_{2}^{1}(Q ; \mathbb{C})$, из оценок $(2.13)$ получим

$$
\begin{aligned}
& \left\|u_{\varepsilon}-u^{0}\right\|_{L_{2}(Q ; \mathbb{C})} \leqslant c \sqrt{\varepsilon}\left\|\partial_{\bar{z}} f\right\|_{L_{2}(Q ; \mathbb{C})}, \\
& \left\|u_{\varepsilon}-u_{1}^{\varepsilon}\right\|_{W_{2}^{1}(Q ; \mathbb{C})} \leqslant c \sqrt{\varepsilon}\left\|\partial_{\bar{z}} f\right\|_{L_{2}(Q ; \mathbb{C})}
\end{aligned}
$$


(Здесь и ниже $c>0$ постоянная, независящая от $\varepsilon$ и $f$.)

Так как $u_{\varepsilon}, u^{0}$ - решения краевых задач Римана-Гильберта, имеем $u_{\varepsilon}=$ $A_{\varepsilon}^{-1} f, u^{0}=A_{0}^{-1} f$. Следовательно, ввиду (3.1), получим

$$
\left\|\left(A_{\varepsilon}^{-1}-A_{0}^{-1}\right) f\right\|_{L_{2}(Q ; \mathbb{C})} \leqslant c \sqrt{\varepsilon}\left\|\partial_{\bar{z}} f\right\|_{L_{2}(Q ; \mathbb{C})}, \quad f \in W_{0}(Q) .
$$

Отсюда, так как отображение $\partial_{\bar{z}}: W_{0}(Q) \rightarrow L_{2}(Q ; \mathbb{C})$ есть изоморфизм, имеем

$$
\left\|\left(A_{\varepsilon}^{-1}-A_{0}^{-1}\right) \partial_{\bar{z}}^{-1} g\right\|_{L_{2}(Q ; \mathbb{C})} \leqslant c \sqrt{\varepsilon}\|g\|_{L_{2}(Q ; \mathbb{C})}, \quad g \in L_{2}(Q ; \mathbb{C}) .
$$

Следовательно,

$$
\left\|\left(A_{\varepsilon}^{-1}-A_{0}^{-1}\right) \partial_{\bar{z}}^{-1}\right\|_{L_{2}(Q ; \mathbb{C}) \rightarrow L_{2}(Q ; \mathbb{C})} \leqslant c \sqrt{\varepsilon} .
$$

Значит, оценка (2.14) доказана.

Ввиду того, что имеет место вложение $W_{0}(Q) \subset L_{2}(Q ; \mathbb{C})$, операторы $A_{\varepsilon}^{-1}$, $A_{0}^{-1}$ можно рассматривать как операторы, действующие из $W_{0}(Q)$ в $L_{2}(Q ; \mathbb{C})$, тогда из оценки (3.1) получим (2.15).

Аналогично (3.3) из (3.3) и (2.11) имеем

$$
\begin{aligned}
\|\left(A_{\varepsilon}^{-1}-A_{0}^{-1}\right) & \partial_{\bar{z}}^{-1} g-\varepsilon\left(N \partial_{z} A_{0}^{-1} \partial_{\bar{z}}^{-1} g+\right. \\
& \left.+M \partial_{\bar{z}}^{-1} \overline{A_{0}^{-1} \partial_{\bar{z}}^{-1} g}\right)\left\|_{W_{2}^{1}(Q ; \mathbb{C})} \leqslant \sqrt{\varepsilon}\right\| g \|_{L_{2}(Q ; \mathbb{C})}, \quad g \in L_{2}(Q ; \mathbb{C}) .
\end{aligned}
$$

Отсюда следует оценка (2.16).

Для завершения доказательства теоремы 2.5 осталось заметить, что (2.17) получается аналогично (2.15), используя оценку (3.2).

\section{Список литературы}

[1] Жиков В.В., Козлов С.М., Олейник О.А. Усреднение дифференциальных операторов. М.: Наука. 1993.

[2] Бирман М.Ш., Суслина Т.А. Периодические дифференциальные операторы второго порядка. Пороговые свойства и усреднения // Алгебра и анализ. 2003. Том 15, вып. 5. С. 1-108.

[3] Жиков В.В. Об операторных оценках в теории усреднения // Доклады РАН. 2005. Том 403, вып. 3. С. 305-308.

[4] Жиков В.В., Пастухова В.В. Об операторных оценках в теории усреднения // Успехи матем. наук. 2016. Том 71, вып. 3. С. 64-73.

[5] Векуа И.Н. Обобщенные аналитические функции. М.: Наука. 1988.

[6] Сиражудинов М.M. O G-сходимости и усреднении обобщенных операторов Бельтрами // Матем. сб. 2008. Том 199, вып. 5. С. 124-155.

[7] Сиражудинов М.М., Сиражудинов P.M. O G-сходимости систем обобщенных операторов Бельтрами // Труды МИАН. 2008. Вып. 121. С. 268-276.

[8] Сиражудинов М.M. O G-компактности одного класса эллиптических систем первого порядка // Дифф. уравн. 1990. Том 26, вып. 2. С. 298-305.

[9] Сиражудинов М.М. О краевой задаче Римана-Гильберта // Дифф. уравн. 1989. Том 25, вып. 8. С. 1400-1406.

[10] Сиражудинов М.М. Асимптотический метод усреднения обобщенных операторов Бельтрами // Матем. сб. 2017. Том 208, вып. 4. С. 111-128.

М. М. Сиражудинов (M. М. Sirazhudinov)

Поступила в редакцию ДНЦ РАН, ДГУ, ДГУНХ

17.02 .2017

E-mail: sirazhmagomed@yandex.ru 